Eine Analyse der dritten Handelsperiode des Emissionshandels

\section{Klimaschutz in Europa: Effektiv, effizient, gerecht}

Nach gemischten Erfahrungen in den ersten Phasen des EU-Emissionshandelssystems werden ab der in diesem Jahr beginnenden dritten Phase tief greifende Änderungen im Regelwerk wirksam. Im November 2012 legte die EU-Kommission außerdem Vorschläge vor, um das System zusätzlich zu verschärfen. Aber machen diese Änderungen den europäischen Klimaschutz tatsächlich effektiver, effizienter und gerechter? Von Achim Lerch und Sven Rudolph

E tgegen der idealistischen Vorstellung der Umweltökonomik vom schlanken Zertifikatshandel sind in Emissionshandelssystemen (EHS) der klimapolitischen Praxis eine Vielzahl detaillierter Ausgestaltungsentscheidungen zu treffen. Aus einer Bewertung auf der Basis von Kriterien der ökologischen Effektivität, der ökonomischen Effizienz und der sozialen Gerechtigkeit lassen sich folgende Empfehlungen für die wichtigsten Elemente eines nachhaltigen EHS formulieren (Rudolph/Lerch et al. 2012).

\section{Nachhaltigkeitsökonomische Bewertung}

Demnach sollte aus nachhaltigkeitsökonomischer Perspektive die Teilnahme für relevante Emittenten verpflichtend sein und der Anwendungsbereich alle wichtigen Schadstoffe und Emittenten umfassen, die Emissionsgesamtmenge sollte knapp und an den ökologischen Notwendigkeiten orientiert sein, eine zusätzliche stetige Verknappung des Angebots an Emissionslizenzen hält unter anderem Innovationsanreize aufrecht und trägt zur Erreichung von Umverteilungsgerechtigkeit bei. Die Erstvergabe sollte per Auktion erfolgen, da diese auch unmittelbar Preissignale setzt und die entstehenden Einnahmen für gerechtigkeitsfördernde Ausgleichsmaßnahmen zur Verfügung stehen. Diese Einnahmen können eingesetzt werden zum Beispiel im Sinne der intranationalen Gerechtigkeit mittels Rückvergütung oder Energiekos- tenzuschuss, im Sinne der internationalen Gerechtigkeit durch Finanztransfers in Entwicklungsländer oder Projektförderung sowie im Sinne der intergenerationellen Gerechtigkeit im Rahmen von Maßnahmen zur Erhöhung der ökologischen Effektivität wie beispielsweise Klimaschutzmaßnahmen. Markteingriffe sollten eng begrenzt werden, um das Preissignal nicht zu verzerren.

\section{Neuerungen im Euro - päischen Emissionshandel}

Nachdem das Emissionshandelssystem der Europäischen Union (EU-EHS) in den ersten Phasen verpflichtend $\mathrm{CO}_{2}$ Emissionen der Stromerzeuger und der energieintensiven Industrie begrenzte, sollen neben dem Luftverkehr ab 2013 weitere Treibhausgasemissionen einbezogen werden, zum Beispiel StickstoffoxidEmissionen aus der chemischen Industrie und perfluorierte Kohlenwasserstoffe aus der Aluminiumproduktion. Für strukturelle Veränderungen stellt die Kommission eine Erweiterung des Anwendungsbereichs auf zusätzliche Sektoren zur Diskussion, wie den von konjunkturellen Schwankungen weniger betroffenen Verkehrssektor.

Aus umweltökonomischer Sicht ist die beschlossene Erweiterung positiv zu bewerten, da so neue Effizienzpotenziale erschlossen und ein größerer Anteil relevanter Gase und Prozesse in den Klimaschutz einbezogen werden. Für den aktuellen Vorschlag der Kommission gilt
Analoges. Auch wenn damit noch immer nicht alle klimarelevanten Aktivitäten einbezogen sind, wird so auch die Verursachungsgerechtigkeit befördert, zumal zu berücksichtigen ist, dass in der EU andere Sektoren wie der Haushaltsund Verkehrssektor teilweise durch andere klimapolitische Instrumente wie Effizienzstandards für Gebäude und Kraftstoffsteuern erfasst werden. Erfahrungen mit einem Emissionshandel für Gebäude in Tokio und Vorschläge aus der Forschung für ein Modell handelbarer Flottenverbrauchsstandards haben aber gezeigt, dass der Emissionshandel auch für andere Sektoren erfolgreich genutzt werden kann.

Nachdem zunächst jeweils nationale Emissionsbudgets galten und die Zertifikate anhand nationaler Allokationspläne (NAP) verteilt wurden, gilt ab 2013 ein gesamteuropäisches Budget, das auf den mittleren Wert des Zeitraums 2008-2012 festgelegt wurde und danach jährlich um 1,76 Prozent verringert wird. Dies ergibt eine Reduktion der Emissionen um 21 Prozent im Jahr 2020 gegenüber 2005. Um die derzeit niedrigen Preise zu erhöhen, hat die Kommission zusätzlich vorgeschlagen, die Anzahl der zu versteigernden Zertifikate in den Jahren 2013 bis 2015 um 900 Millionen zu reduzieren und diese zu einem späten Zeitpunkt in der dritten Handelsperiode wieder in den Markt zu geben. Für strukturelle Veränderungen wurden drei Optionen zur Diskussion gestellt: Die Verschärfung des EU-Reduktionsziels für 2020 auf 30 Prozent mit einer entsprechenden Kürzung der Emissionsgesamtmenge, die endgültige Stilllegung von Phase-III-Zertifikaten und eine frühe Anpassung des linearen Reduktionspfades an das 30Prozent-Ziel.

Aus umweltökonomischer wie aus gerechtigkeitstheoretischer Sicht ist die beschlossene Verringerung der Emissionsgesamtmenge zu begrüßen. Eine dauerhafte Stilllegung dürfte dabei für Planungssicherheit und ein stabiles Preissignal sorgen. Die Verlegung der Mengenfixierung auf die EU-Ebene verringert zudem Administrationskosten und Wettbewerbsverzerrungen. Im April 2013 
wurden die Vorschläge der Kommission jedoch vom EU-Parlament abgelehnt.

Nachdem bis 2012 mindestens 95 Prozent beziehungsweise 90 Prozent der Lizenzen kostenlos an die Unternehmen vergeben wurden, werden ab 2013 mindestens 50 Prozent der Zertifikate versteigert, ab 2027 sogar 100 Prozent. Stromerzeuger müssen bereits ab 2013 alle Zertifikate käuflich erwerben. Allerdings gibt es vielfältige Ausnahmen für energieintensive Branchen. Für den Anteil kostenloser Zuteilung werden auf europäischer Ebene einheitliche Regeln vorgegeben.

Durch die Versteigerung werden Einnahmen generiert, von denen mindestens 20 Prozent für Maßnahmen zur Bekämpfung des Klimawandels in Europa und in Entwicklungsländern eingesetzt werden. Damit werden sowohl ökologische als auch Ziele der intergenerationellen wie der internationalen Gerechtigkeit verfolgt. Unklar bleibt jedoch, was mit den restlichen 80 Prozent der Einnahmen passieren wird und es gibt bislang keinen Mechanismus zum Ausgleich von Problemen der sozialen Gerechtigkeit aufgrund steigender Energiepreise wie etwa eine Pro-Kopf-Rückvergütung. Zusammen mit den Ausnahmen für die energieintensive Industrie muss dies aus Gerechtigkeitsperspektive als ein Hauptkritikpunkt am EU-EHS gelten. Aus ökonomischer Sicht ist zu empfehlen, zumindest Teile des Aufkommens zur Senkung verzerrender Abgaben zu nutzen, um so eine doppelte Dividende zu erzielen.

\section{Den Preisverfall umkehren}

Die Anerkennung von Projektkrediten aus CDM- und JI-Projekten ist grundsätzlich möglich, ab 2013 allerdings begrenzt auf maximal 50 Prozent der in der EU von 2008 bis 2020 durchzuführenden Emissionssenkungen. Um den aktuellen Preistrend nach unten umzukehren schlägt die EU-Kommission als strukturelle Veränderungen eine engere Begrenzung oder ein zeitweises Aussetzen der Anerkennung internationaler Emissionsgutschriften vor.
Aus nachhaltigkeitsökonomischer Perspektive ist insbesondere die beschlossene Erhöhung des Versteigerungsanteils positiv zu bewerten. Sie sorgt für sofortige verlässliche Preissignale, reduziert Wettbewerbsverzerrungen und dient der Verursachungsgerechtigkeit. Eine quantitative Begrenzung der Anerkennung von Projektkrediten mindert zwar Effizienzpotenziale, sorgt aber andererseits für stärkere heimische Innovationsanreize. Vorteilhaft wäre zudem aus ökologischer Sicht die Beschränkung auf verlässliche Projekte, beispielsweise Gold-standardGutschriften.

Ab 2013 sind Markteingriffe wie Reserveverkäufe geplant, wenn der Preis einer Periode mehr als das Dreifache der Vorperiode beträgt. Die derzeit allerdings eher zu niedrigen Preise könnten nach einem Kommissionsvorschlag zur strukturellen Überarbeitung des EU-Emissionshandels entweder durch eine Preisuntergrenze oder durch das Befüllen der Preisbegrenzungsreserve aus zurückgehaltenen Zertifikaten erhöht werden. Solche Markteingriffe sind allerdings nicht nur aus Effizienzgründen, sondern auch aus der Perspektive der Verursachungsgerechtigkeit kritisch zu sehen. Preiskorridore sind mit Vorsicht zu genießen, da sie gerade im Falle von Höchstpreisen zu Fehlallokationen führen können. Mindestpreise können aber, so lehren die Erfahrungen aus dem Emissionshandelssystem im Nordosten der USA, aus ökologischer und gerechtigkeitstheoretischer Sicht selbst bei einem sehr schwachen Emissionsminderungsziel dafür sorgen, dass Aufkommen generiert wird, das für den Klimaschutz oder soziale Ausgleichsmaßnahmen genutzt werden kann.

\section{Fazit}

Die Analyse des EU-Emissionshandels zeigt, dass mit Beginn der dritten Handelsperiode zahlreiche Veränderungen in Kraft treten, die aus nachhaltigkeitsökonomischer Sicht überwiegend positiv zu beurteilen sind. Hinsichtlich der aktuellen Vorschläge der Kommission wäre sicherlich eine permanente Kürzung der Emissionsmenge eine vielversprechende
Maßnahme zur Erhaltung von Innovationsanreizen im europäischen Klimaschutz gewesen.

Kritisch zu sehen ist dagegen die Etablierung einer Kostenbeschränkungsreserve mit potenziellen Preisobergrenzen. Ein Hauptkritikpunkt aus Gerechtigkeitssicht ist, dass kein Mechanismus zum Ausgleich sozialer Gerechtigkeitsprobleme aufgrund steigender Energiepreise vorgesehen ist. Gerade für die EU, die infolge der Finanz- und Schuldenkrise ohnehin mit erheblichen sozialen Verwerfungen zu kämpfen hat, könnte der Emissionshandel daher aufgrund künftig ambitionierterer Reduktionsziele und steigender Energiepreise Probleme der sozialen Gerechtigkeit noch verstärken. Für Deutschland, wo die angestrebte Energiewende bereits aktuell zu erheblichen Strompreiserhöhungen und entsprechenden sozialen Folgen führt, gilt dies in besonderem Maße.

\section{Literatur}

EU-Commission (2012): Report from the Commission to the European Parliament and the Council - The state of the European carbon market in 2012. Brüssel.

Rudolph, S./Lerch, A. et al. (2012): Towards sustainable carbon markets - requirements for effective, efficient, and fair emission trading schemes. In: Kreiser, L. et al. (Eds.): Carbon Pricing, Growth, and the Environment, Critical Issues in Environmental Taxation, Band XI, Cheltenham/Northampton: Edward Elgar, S. $167-183$

\section{AUTOREN + KONTAKT}

Dr. Achim Lerch ist Professor für Allgemeine Volkswirtschaftslehre an der Hessischen Berufsakademie/University of Cooperative Education.

E-Mail: achim.lerch@hessische-ba.de

Dr. Sven Rudolph ist wissenschaftlicher Assistent am Competence Center for Climate Change Mitigation and Adaptation (CliMA) der Universität Kassel.

E-Mail: s.rudolph@wirtschaft.uni-kassel.de Beide waren Mitarbeiter im Forschungsprojekt LETSCaP zur internationalen Verknüpfung von Emissionshandelssystemen, das vom Bundesministerium für Bildung und Forschung (BMBF) gefördert wurde. 
Copyright (C) 2013, IÖW und oekom Verlag. Die Nutzung des Artikels ist Abonnenten von Ökologisches Wirtschaften vorbehalten. Nachdruck und Vervielfältigung des Artikels einschließlich Speicherung und Nutzung auf optischen und elektronischen Datenträgern nur mit Zustimmung der Redaktion von Ökologisches Wirtschaften (http://www.oekologischeswirtschaften.de). 